\title{
Regional odontodysplasia - a rare developmental dental anomaly
}

\author{
Kacper Nijakowski, Anna Surdacka \\ Department of Conservative Dentistry and Endodontics, Poznan University of Medical Sciences, Poznan, Poland
}

\section{ABSTRACT}

Regional odontodysplasia is a rare developmental dental anomaly affecting ectodermal and mesodermal tissues. Hypocalcification and hypoplasia are most often observed in one quadrant. The aetiology remains uncertain - the harmful local factor in the perinatal period is considered. It occurs more often in the maxilla, with a predisposition to females. The report presents a clinical case of a 5-year-old boy with regional odontodysplasia affecting deciduous and permanent dentition in the left quadrant of the mandible. The radiological image revealed characteristic "ghost teeth" in primary dentition and poorly developed buds of secondary teeth. Because of recurrent purulent inflammations, his milk teeth were surgically removed. In the literature, there is no consensus on the treatment of choice. However, it is suggested that affected teeth should not be extracted until inflammation occurs to allow the development of surrounding bone. Due to the age-related functional and aesthetic needs, the patients require long-term specialist care.

\section{KEY WORDS:}

case report, deciduous teeth, dental anomaly, ghost teeth, regional odontodysplasia.

\section{INTRODUCTION}

Regional odontodysplasia is a rare developmental dental anomaly affecting ectodermal (enamel) and mesodermal tissues (dentine, cementum, pulp), mostly in one quadrant. The term "odontodysplasia" was first used by Zegarelli et al. in 1963, and the prefix "regional" was added by Pindborg in 1970 [1-3]. Epidemiologic reports are rare - only 174 cases of regional odontodysplasia have been published in English up to January 2021. Its prevalence is estimated at less than $1: 1,000,000$. Hypocalcification and hypoplasia of dental hard tissues involve both primary and permanent dentitions - in nearly half of the patients. This condition occurs more often in the maxilla (approximately twice), with a slight female predilection [1-3].

The aetiology of the disease remains unknown. The potentially harmful local factor in the perinatal period is considered. In the literature, the possible causal fac- tors such as disorders of neural crest cell migration, local trauma or ischaemia, vascular defects, teratogenic medications, radiation, hyperpyrexia, activation of latent viruses, Rh incompatibility, and nutritional or metabolic deficiencies are mentioned $[1,2,4]$.

This case report presents the classical clinical and radiological appearance of regional odontodysplasia. According to our knowledge, this is the first case of odontodysplasia described in Poland.

\section{CASE REPORT}

A 5-year-old male patient was referred to our Department by concerned parents. The chief complaint was recurrent purulent inflammations of deciduous teeth in the left mandibular region. According to his father, these teeth had been different from the others because of their yellowish-brown colour and tendency to crumble rapidly. The medical and family histories were unremarkable.

\section{ADDRESS FOR CORRESPONDENCE:}

Kacper Nijakowski, Department of Conservative Dentistry and Endodontics, Poznan University of Medical Sciences, Bukowska 70, 60-812 Poznan, Poland, e-mail: kacpernijakowski@ump.edu.pl 


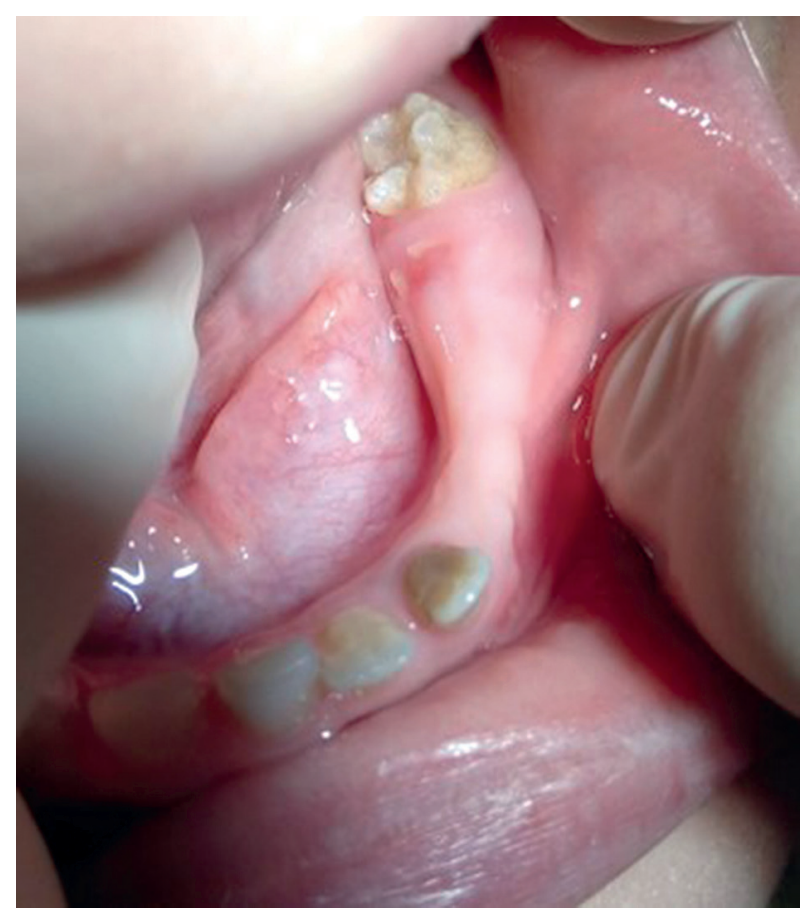

FIGURE 1. Intraoral view of the affected mandibular left quadrant (4 years 9 months)

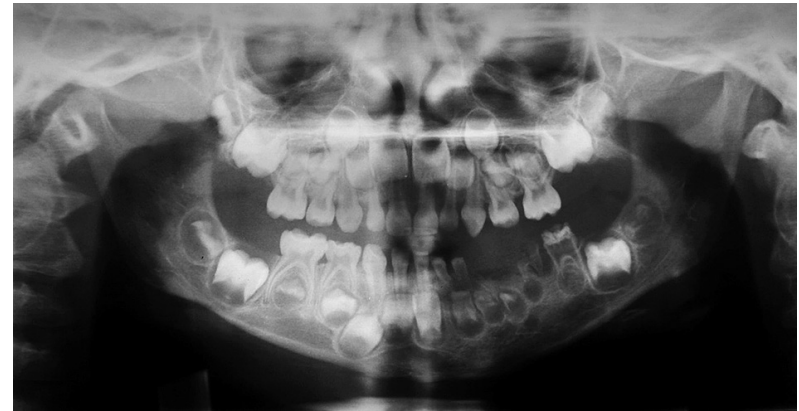

FIGURE 2. Panoramic radiograph presenting the teeth with "ghostlike" appearance in the mandibular left quadrant (4 years 6 months)

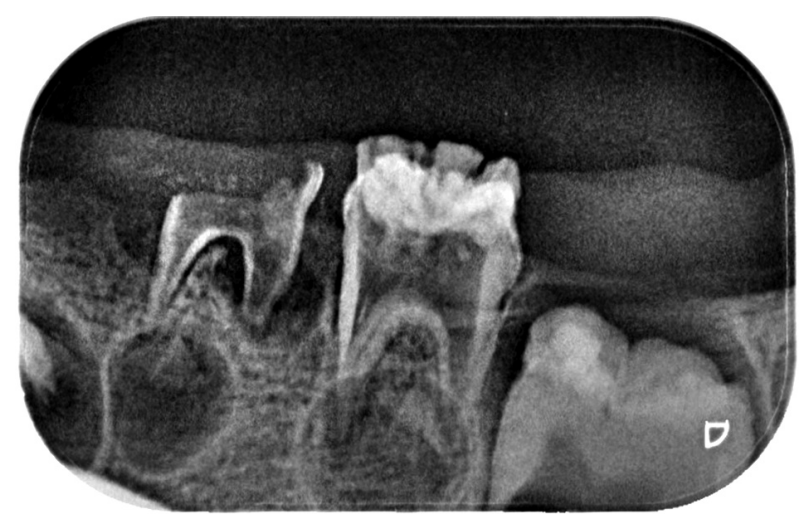

FIGURE 3. Periapical radiograph presenting the primary molars with "ghost-like" appearance (4 years 9 months)

The mother had a normal pregnancy and delivery. Both parents reported no previous history of tooth or genetic anomalies on either side of the family. The two siblings (elder sister and younger brother) also did not have any quantitative or qualitative dental disorders.

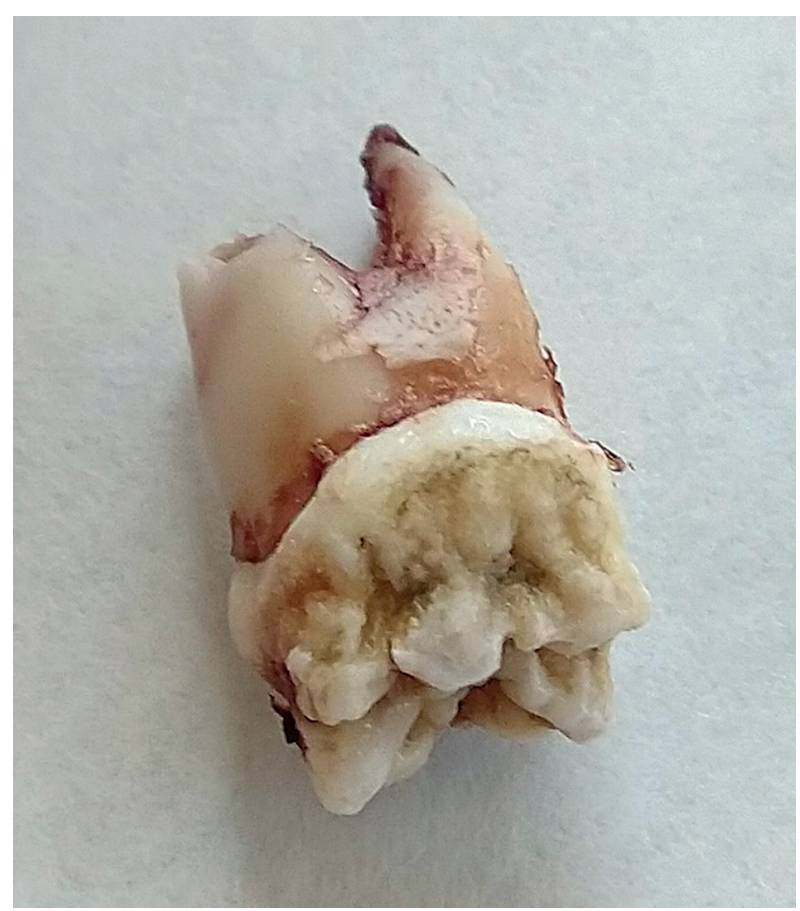

FIGURE 4. Extracted primary mandibular left second molar with signs of enamel hypocalcification and hypoplasia

The extraoral examination revealed a slight asymmetry in the lower part of the face. The intraoral examination confirmed gingival swelling on the left side of the mandible, which was painful upon palpation (Fig. 1). Present primary mandibular left teeth manifested abnormal morphology with irregular hypoplastic surface and yellowish-brown colour, and no signs of caries. The canine in this quadrant had been extracted 6 months earlier due to purulent inflammation. Other teeth did not show any pathology, except the primary maxillary right central incisor with bluish colouring of the crown caused by aseptic necrosis after trauma when the patient was 18 months old.

A panoramic radiograph (Fig. 2) had been taken 3 months before the boy came for the appointment. Radiographically, the mandibular left deciduous teeth and the follicles of succedaneous permanent teeth presented "ghost-like" appearance. All the primary teeth and the germs of the permanent teeth in other quadrants were age-appropriately developed. Also, the noticeable underdevelopment of the left side of the mandible and the slight shift of the midline to the left were observed.

The periapical radiographs (Fig. 3) of the affected teeth demonstrated blurred demarcation between very thin layers of enamel and dentin. Moreover, visible wide pulp chambers with open apices were characteristic features indicative of "ghost teeth".

The treatment suggested to the patient's father was the surgical removal of the residual roots of the primary mandibular left first molar. After a week on a follow-up visit, despite the absence of caries, an active fistula and increased mobility were found in the primary left incisors. 
These affected teeth were also extracted. After a month, due to severe pain during biting, it was decided to remove the last deciduous tooth in this quadrant (Fig. 4).

Due to the early loss of primary teeth in the left mandibular quadrant (Fig. 5), the patient was referred for the orthodontic consultation. A removable acrylic partial denture was planned, whereas in case of expected deficiencies in secondary dentition also implantoprosthetic treatment would be considered after the growth period.

Two years after the first visit, a follow-up panoramic radiograph was taken (Fig. 6), which presented asymmetry due to the underdeveloped left half of the mandible as well as slight progress in the development of the permanent teeth in the affected quadrant.

\section{DISCUSSION}

The differential diagnosis of regional odontodysplasia should include other developmental dental disorders, such as dentin dysplasia, dentinogenesis imperfecta, amelogenesis imperfecta, or hypophosphatasia [4].

Patients with regional odontodysplasia need a longterm multidisciplinary specialist approach $[2,4,5]$. The role of paediatricians is to refer them to the dental office as soon as possible to increase the chance of keeping teeth for a longer time in the oral cavity. In the literature, there is no consensus on the treatment of choice. It is suggested that affected teeth without periapical inflammation should be retained to allow the development of surrounding bone. However, in case of the development of acute and exacerbated abscesses, immediate tooth extraction is recommended $[2,4]$. Then, edentulous areas should be restored with removable acrylic appliances to maintain aesthetic and masticatory functions. Also, it is essential to lessen the psychological effects of premature tooth loss. As an alternative treatment method, some clinicians reported the autotransplantation of the permanent premolars from the unaffected side with the dentoalveolar discrepancy $[3,5]$. Moreover, in adulthood, implantoprosthetic treatment is advisable.

The treatment of odontodysplasia should be considered based on the degree and extent of the disorder, as well as the patient's age, attitude towards dental treatment, or their age-related functional and aesthetic needs [5].

\section{CONCLUSIONS}

Regional odontodysplasia is a unique developmental dental anomaly resulting in severe morphological and functional disorders of the masticatory system.

Despite the absence of signs of caries, affected teeth may cause the development of recurrent purulent inflammations in the form of life-threatening abscesses or fistulas.

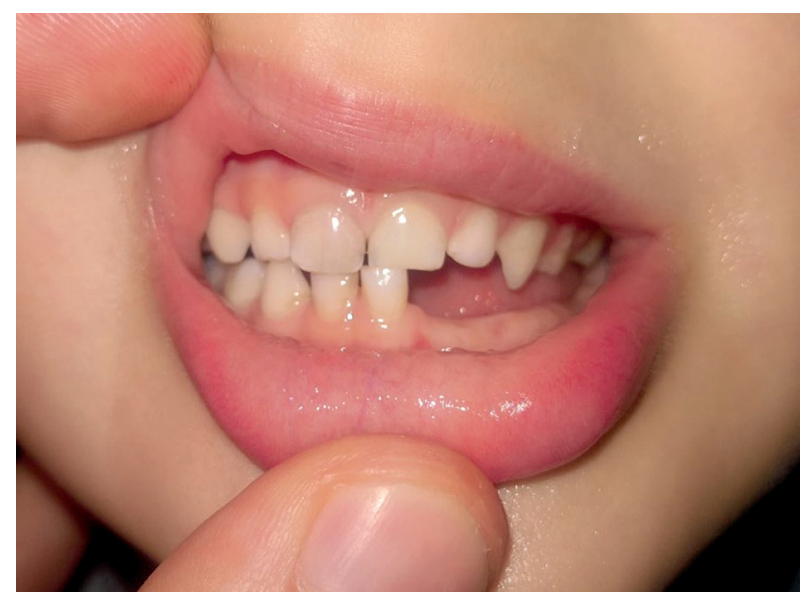

FIGURE 5. Extraoral view of the occlusion after extractions of affected primary mandibular left teeth ( 5 years)

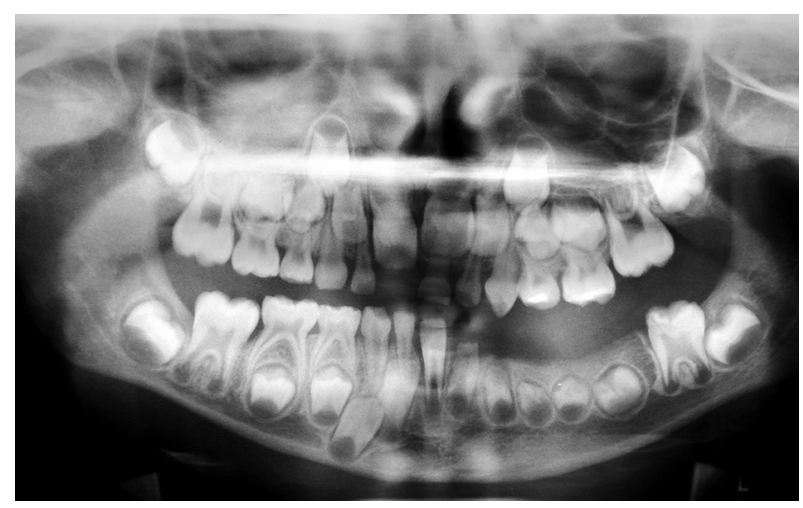

FIGURE 6. Follow-up panoramic radiograph presenting the follicles of succedaneous permanent teeth in the mandibular left quadrant (6 years 8 months)

Its treatment requires multidisciplinary cooperation between paedodontists, surgeons, orthodontists, and prosthetists.

\section{DISCLOSURE}

The authors declare no conflict of interest.

\section{REFERENCES}

1. Alotaibi O, Alotaibi G, Alfawaz N. Regional odontodysplasia: An analysis of 161 cases from 1953 to 2017. Saudi Dent J 2019; 31: 306-310.

2. Rashidian A, Afsharian Zadeh M, Azarshab M, Zarrabian T. Regional Odontodysplasia: Report of a case. J Dent (Shiraz) 2013; 14: 197-200.

3. Ziegler S, Neukam FW. Regional odontodysplasia: Orthodontic treatment and transplantation of premolars. Am J Orthod Dentofacial Orthop 2012; 142: 710-719.

4. Murthy P, Deshmukh S. Regional odontodysplasia and its treatment maneuver. Int J Health Allied Sci 2013; 2: 153-158.

5. Cahuana A, González Y, Palma C. Clinical management of Regional Odontodysplasia. Pediatr Dent 2005; 27: 34-39. 
